\title{
Severe SARS-CoV-2 infection is defined by a shift in the serum lipidome resulting in dysregulation of eicosanoid lipid immune mediators
}

\section{Benjamin Schwarz}

National Institute of Allergy and Infectious Diseases

\section{Lokesh Sharma}

Yale University

\section{Lydia Roberts}

National Institute of Allergy and Infectious Diseases

\section{Xiaohua Peng}

Yale University

Santos Bermejo

Yale University

lan Leighton

National Institute of Allergy and Infectious Diseases

\section{Arnau Casanovas-Massana}

Yale University https://orcid.org/0000-0002-3301-6143

Shelli Farhadian

Yale University

\section{Albert Ko}

Yale School of Public Health https://orcid.org/0000-0001-9023-2339

\section{Yale IMPACT Team}

Yale University

\section{Charles Dela Cruz}

Yale University School of Medicine

Catharine Bosio ( $\sim$ bosioc@niaid.nih.gov )

National Institute of Allergy and Infectious Diseases

\section{Short Report}

Keywords:

Posted Date: July 22nd, 2020

DOI: https://doi.org/10.21203/rs.3.rs-42999/v1 
License: (c) (i) This work is licensed under a Creative Commons Attribution 4.0 International License. Read Full License 


\title{
Title
}

Severe SARS-CoV-2 infection in humans is defined by a shift in the serum lipidome resulting in dysregulation of eicosanoid immune mediators

Benjamin Schwarz ${ }^{1 \&}$, Lokesh Sharma ${ }^{2 \&}$, Lydia Roberts ${ }^{1 \&}$, Xiaohua Peng $^{2}$, Santos Bermejo ${ }^{2}$, Ian Leighton $^{1}$, Arnau Casanovas Massana ${ }^{3}$, Shelli Farhadian ${ }^{4}$, Albert I. Ko ${ }^{3}$, Yale IMPACT Team, Charles S. Dela Cruz ${ }^{2 \$^{*}}$, Catharine M. Bosio ${ }^{1 \$^{*}}$

${ }^{1}$ Laboratory of Bacteriology, National Institute of Allergy and Infectious Diseases, National Institutes of Health, Hamilton, MT, USA ${ }^{2}$ Section of Pulmonary and Critical Care and Sleep Medicine, Yale University School of Medicine, New Haven, Connecticut ${ }^{3}$ Department of Epidemiology of Microbial Diseases, Yale School of Public Health, New Haven, CT $06520{ }^{4}$ Department of Medicine, Section of Infectious Diseases, Yale University School of Medicine, New Haven, CT, 06520

\&Authors contributed equally

\$Authors contributed equally

*Corresponding authors:

Catharine M Bosio: bosioc@niaid.nih.gov

Charles S. Dela Cruz: Charles.delacruz@yale.edu

\begin{abstract}
Abbreviations
LM-eicosanoid and docosanoid lipid mediators, PE-phosphatidylethanolamine, LPE- lyso-PE, PCphosphatidylcholine, LPC-Iyso-PC, PS-phosphatidylserine, $\mathrm{PE}(\mathrm{O})$ or $\mathrm{PE}(\mathrm{P})$ - plasmenyl or plasmanyl plasmalogen, TAG- triacylglycerol, DAG-diacylglycerol, MAG-monoacylglycerol, CE-cholesterol ester, Cer-
\end{abstract}


ceramide, DCer- dihydroceramide, HCer-hexosylceramide, LCer-lactosylceramide, SM-sphingomyelin, FAC-frees fatty acid, Rv-resolvin, LX-lipoxin, LT-luekotriene, HETE- hydroxyeicosatetraenoic acid, HEPEhydroxyeicosapentaenoic acid, HDHA- hydroxydocosahexaenoic acid, HDPA- hydroxydocosapentaenoic acid, PG-prostaglandin, PD- D-series protectin, TxB2- Thromboxane B2, LC-MS/MS- liquid chromatography tandem mass spectrometry, CBA- cytometric bead array, PCA- principle component analysis, PLSDA- partial least square discriminant analysis 


\section{Introductory Paragraph}

2 The COVID-19 pandemic has affected more than 10 million people worldwide with mortality exceeding

3 half a million patients. Risk factors associated with severe disease and mortality include advanced age,

4 hypertension, diabetes, and obesity. ${ }^{1}$ Clear mechanistic understanding of how these comorbidities

5 converge to enable severe infection is lacking. Notably each of these risk factors pathologically disrupts

6 the lipidome and this disruption may be a unifying feature of severe COVID-19. ${ }^{1-7}$ Here we provide the

7 first in depth interrogation of lipidomic changes, including structural-lipids as well as the eicosanoids and

8 docosanoids lipid mediators (LMs), that mark COVID-19 disease severity. Our data reveal that

9 progression from moderate to severe disease is marked by a loss of specific immune regulatory LMs and

10 increased pro-inflammatory species. Given the important immune regulatory role of LMs, these data

11 provide mechanistic insight into the immune balance in COVID-19 and potential targets for therapy with

12 currently approved pharmaceuticals. ${ }^{8}$ 


\section{Main Text}

15 Lipids function in disease to rearrange cellular signaling structures, modify metabolic processes, absorb

16 reactive species, and act directly as both autocrine and endocrine ligands in the regulation of the

17 immune system. Susceptibility to COVID-19 disease is strongly associated with pre-existing conditions

18 characterized by dysregulation of the lipidome and metabolome. ${ }^{4-6}$ While several studies have examined

19 the systemic metabolic correlates of COVID-19, a well resolved interrogation of the lipidomic changes in

20 COVID-19 severity has not been pursued. ${ }^{9-12}$ To measure lipidomic changes in COVID-19 and gain

21 mechanistic insights into how these changes may drive disease severity, we used serum draws from 19

22 healthy patients (healthy), 18 COVID-19 patients who did not require ICU admission (moderate) and 20

23 patients that required ICU admission (severe). The demographics, preexisting conditions, and treatment

24 details of these patients are indicated in Table 1. Lipid and metabolite measurements were made using

25 a series of targeted LC-MS/MS methods providing high-confidence feature identification. . $^{13,14}$

26 Importantly for lipidomic analysis, this enabled the resolution of acyl-chain length and degree of

27 unsaturation, which are both essential for understanding structural and functional rearrangement of the

28 lipidome.

29 Changes in primarily polar metabolites among COVID-19 patient cohorts from China, Italy, and France

30 have been reported..$^{9-12}$ In agreement with those studies, we observed a dysregulation of amino acid

31 pools, interruption of the glucose to lactate balance, and dysregulation of nucleotide catabolic products

32 such as xanthine, hypoxanthine, and urate (Sup. Fig. 1a-d). ${ }^{9-12}$ These indicators suggest a robust

33 xanthine oxidase stress response, associated with heart disease, ${ }^{15-17}$ and likely reflect the degree of

34 hypoxia/hypoxemia in the patient, which is a known to be associated with COVID-19 mortality. ${ }^{18-22}$ These

35 data also indicate broad agreement across international populations in metabolic correlates of COVID-

3619. 
37 We next measured lipidomic profiles across severe and moderate COVID-19 infection along with the

38 healthy controls. To ensure comprehensive recovery of lipid classes, we utilized a modified chloroform

39 extraction method to recover both neutral and polar lipids. ${ }^{23-25}$ By unbiased principle component

40 analysis (PCA), infected patients segregated from healthy in the negative ionization dataset but

41 overlapped in the positive ionization dataset (Sup. Fig. 2a-d). Group-biased partial least square

42 discriminant analysis (PLSDA) of the combined lipid dataset shows non-overlapping healthy and infected

43 separation across the primary axis of variance and a subgroup of severe patients that separate across

44 the secondary axis of variance (Fig. 1a). Specifically, the infected cohorts were associated with increased

45 levels of free poly-unsaturated fatty acids (PUFAs), rearrangement of certain sphingomyelins, and

46 decreased levels of PUFA-containing plasmalogens (Fig. 1b). Parallel univariate analysis revealed that

47 numerous neutral lipids significantly varied between severe and healthy controls, which may reflect

48 either changes in metabolism during infection or pre-infection differences in lipid levels due to pre-

49 existing conditions such as obesity (Sup. Fig 2e-g). Minor patterns distinguishing both infected from

50 healthy cohorts and moderate from severe disease were observed across lysophospholipids (Sup. Fig.

$512 \mathrm{2h-j),} \mathrm{cholesterol} \mathrm{esters} \mathrm{(chol-est)} \mathrm{(Sup.} \mathrm{Fig.} \mathrm{2k-m),} \mathrm{and} \mathrm{sphingolipids} \mathrm{(Sup.} \mathrm{Fig.} \mathrm{2n-p).}$

52 Across all lipid classes, PUFA containing lipids were abundant amongst the pool significantly varied lipids

53 between COVID-19 patients and healthy controls (Fig. 1c-e). To examine the regulation of PUFAs

54 between lipid classes and patient groups, we categorized lipids containing C20:4, C20:5, C22:5 or C22:6,

55 which likely represent arachidonic acid (AA), eicosapentaenoic acid (EPA), docosapentaenoic acid (DPA),

56 and docosahexaenoic acid (DHA). Of these PUFA-containing families, changes in the C20:4 series were

57 overrepresented in infected cohorts and could distinguish the severe from the moderate disease

58 patients (Fig. 1c-e). Many of these differentially regulated C20:4 species were the same plasmalogen

59 species that drove separation between infected and healthy cohorts by PCA (Sup. Fig. 2b) and PLSDA

60 (Fig. 2b, f-h). Interestingly, the depletion of PUFA-containing plasmalogen and increased levels of the 
61 corresponding free-fatty acids (FAC) indicates the disease progression from the moderate to the severe

62 disease across each PUFA family (Fig. 1i-I). Plasmalogen is known to be a primary pool of PUFAs in both

63 immune and structural cells. ${ }^{2,26}$ Upon systemic immune activation, PUFAs are liberated from their parent

64 glycerolipids and subsequently converted to a wide variety of immune signaling eicosanoids and

65 docosanoids. ${ }^{27-32}$ The balance between pro-inflammatory, immune-regulating, and pro-resolving lipid

66 mediators can drastically change the efficacy of the immune response during infectious and sterile

67 inflammatory diseases as well as during the successful resolution of inflammation following disease. ${ }^{33-35}$

68 Therefore, we assessed the correlation of the eicosanoid and docosanoid species with COVID-19 disease

69 severity. We targeted 67 eicosanoid and docosanoid species using LC-MS/MS and 15 cytokines by

70 Cytometric Bead Array (CBA) or ELISA to relate lipid changes to markers of disease severity. Eicosanoid

71 and docosanoid lipid mediator (LM) signals were assessed by comparison to standards and available

72 spectral libraries (Sup. Fig. 3-5). ${ }^{36}$ PCA analysis of the combined LM and cytokine data showed separation

73 of infected and healthy cohorts and overlapping, but distinct, separation between moderate and severe

74 patients (Fig. 2a). Nearly all LMs measured were positively correlated with infection (Fig. 2b). Univariate

75 analysis showed significant enrichment of the majority of LMs measured in both the moderate and

76 severe groups (Fig. 2c, d). Interestingly, moderate and severe disease were characterized by unique

77 milieus of LMs and cytokines (Fig. 2b, e). Moderate disease was characterized by significantly higher

78 levels of the pro-resolving LM resolvin E3 (RvE3). Further, there was a trend toward increased presence

79 of the prostaglandin family members, particularly PGE2 $(p=0.105)$, PGFD2 $(p=0.220)$ and PGF2a $(p=$

80 0.242). In contrast, severe disease was characterized by a further increase in free PUFAs levels, AA-, EPA-

81 , DPA and DHA-derived mono-hydroxylated species and AA-derived dihydroxylated species (Fig. 2c-e).

82 This shift in specific immune regulatory LMs in severe disease suggests that an imbalance of LMs may

83 contribute to disease progression. LMs are generated by a single or a series of oxygenase mediated

84 conversions of the parent PUFA. To examine the potential contribution of each oxygenase enzyme to 
85 the severe disease phenotype we grouped LMs according to synthesis pathway (Fig. 2g-k). Several LMs

86 are shared between multiple enzyme groups as they require sequential stereospecific hydroxylations.

87 This grouping revealed that moderate disease was characterized by higher cyclooxygenase activity (COX)

88 as well as certain products of ALOX12 while severe disease is characterized by greater activity of ALOX5

89 and cytochrome p450 (CYP) enzymes. This is good agreement with previous observations from

90 influenza, which associated symptom severity with ALOX5 activity. ${ }^{37}$

91 COVID-19 comorbidities including obesity, age, heart disease, and diabetes are characterized by

92 dysregulation of the homeostatic lipidome. ${ }^{1,3}$ To assess the correlation of these conditions with the

93 shifts in LM pools and their glycerolipid precursors, we overlaid age, sex, BMI, diabetes, heart disease,

94 survival, and antiviral treatment onto the separate PCAs (Sup. Fig. 7-15). Age and sex were evenly

95 distributed across the infected cohort on all PCAs (Sup. Fig. 7, 8). Of the treatments examined, only

96 remdesivir showed a negative correlation with the disease severity (Sup. Fig. 9-11). BMI, diabetes, heart

97 disease and morbidity segregated with the severity of disease across all datasets (Sup. Fig. 12-15). From

98 this study, it is likely that the lipidomic imbalance associated with severe disease is at least partially a

99 consequence of homeostatic disruption of the lipidome due to these pre-existing conditions. It is likely

100 that these pre-existing lipidomic imbalances are further exacerbated during COVID-19 through

101 dysregulation of the LM response resulting in severe disease, impaired resolution and persistent

102 inflammation.

103 Elevation of ALOX5- and CYP-dependent LMs in severe COVID-19 patient sera suggested systemic

104 upregulation of these pathways. To examine the cellular origin of these enzymes in COVID-19 patients,

105 we interrogated a published single cell RNAseq dataset of COVID-19 patient PBMCs for expression of

106 ALOX and CYP genes (Sup. Fig. 6a, b).$^{38}$ ALOX5 expression was detected in most of the 20 cell types

107 identified (Fig. 3a-b) with the highest expression in CD14 monocytes, CD16 monocytes, neutrophils, B

108 cells, and DCs (Fig. 3c). ALOX5 expression was significantly increased in neutrophils and trended upward 
109 in CD14 monocytes, CD16 monocytes, and developing neutrophils (a population found almost

110 exclusively in diseased individuals) from COVID-19 patients compared to healthy controls. Interestingly,

111 severe COVID-19 is characterized by elevated ALOX5 expressing monocyte/macrophage population and

112 depletion of lymphocyte populations. ${ }^{38-40}$ The absence of CYP genes in the blood was consistent with

113 the primarily hepatic localization of these enzymes. ${ }^{41}$ These data suggest a systemic dysregulation of

114 ALOX5 and further support the metabolic dysregulation of the liver in severe disease. ${ }^{42}$

115 


\section{Conclusion}

117 These results provide the first detailed lipidomic understanding of COVID-19 disease progression and

118 represent one of the first combinations of bulk lipidomic and eicosanoid data to map mobilization of

119 lipids in human infectious disease. ${ }^{7,37}$ We provide evidence that a systemic lipid network consisting of

120 liberated PUFAs from plasmalogen and their subsequent conversion to LMs, capable of modulating

121 inflammatory responses, characterizes both the onset and severity of COVID-19. Specifically, the loss of

122 the immune regulatory prostaglandins and the increased production of AA-derived products of ALOX5

123 and cytochrome P450 provides both a measure of disease severity and a mechanistic understanding of

124 the immune balance allowing for patient recovery. ${ }^{43}$ Importantly, these pathways are directly

125 targetable with drugs previously approved for use in other inflammatory conditions and, thus, provide

126 therapeutic opportunities to control severe COVID-19. 27,31

127 
129 We are deeply indebted to the patients and families of patients for their contribution to this study. Prof.

130 Charles N. Serhan and his group including, K. Boyle, A. Shay, C. Jouvene, X. de la Rosa, S. Libreros, and N.

131 Chiang generously provided methodology, consultation and extensive training for the assessment of

132 lipid mediators by LC-MS/MS. AB Sciex in particular M. Pearson, P. Norris and P. Baker (currently Avanti

133 Polar Lipids) provided LC-MS/MS consultation and methods. Lokesh Sharma is supported by Parker B

134 Francis Fellowship. Charles Dela Cruz is supported by Veterans affairs Merit Grant (BX004661) and

135 Department of Defense grant (PR181442). Albert Ko and Charles Dela Cruz are supported by a U19

136 supplement for this work (A1089992-09S2). This work was supported by the Intramural Research

137 Program of the National Institutes of Health, National Institute of Allergy and Infectious Diseases. This

138 work was supported by the Department of Internal Medicine at the Yale School of Medicine, Yale School

139 of Public Health, and the Beatrice Kleinberg Neuwirth Fund.

\section{Author Contributions}

142 B.S., L.S., C.D.C and C.M.B. conceived of experiment. L.S., X.P., S.B., A.C.M, S. F. and A.I.K and the Yale 143 IMPACT Team enrolled patients and collected samples. B.S., L.R. and I.L. extracted samples and collected

144 data. B.S. conducted metabolomics and lipidomics analysis. L.R. conducted single cell RNAseq analysis 145 and cytokine analysis. B.S., L.R., L.S., C.D.C. and C.M.B wrote the manuscript. Yale Impact team: (Listed in 146 alphabetical order) Kelly Anastasio, Michael H. Askenase, Maria Batsu, , Sean Bickerton, Kristina Brower, 147 Molly L. Bucklin, Staci Cahill, , Yiyun Cao, Edward Courchaine, , Giuseppe Deluliis, John Fournier, Bertie 148 Geng, Laura Glick, Akiko Iwasaki, Nathan Grubaugh, Chaney Kalinich, William Khoury-Hanold, Daniel 149 Kim, Lynda Knaggs, Maxine Kuang, Eriko Kudo, Joseph Lim, Melissa Linehan, Alice Lu-Culligan, , Anjelica 150 Martin, Irene Matos, David McDonald, Maksym Minasyan, M. Catherine Muenker, Nida Naushad, Allison 151 Nelson, Jessica Nouws, , Abeer Obaid, Camilla Odio, Saad Omer, Isabel Ott, Annsea Park, Hong-Jai Park, 
152 Mary Petrone, Sarah Prophet, Harold Rahming, Tyler Rice, Kadi-Ann Rose, Lorenzo Sewanan, Denise

153 Shepard, Erin Silva, Michael Simonov, Mikhail Smolgovsky, , Nicole Sonnert, Yvette Strong, Codruta

154 Todeasa, Jordan Valdez, Sofia Velazquez, Pavithra Vijayakumar, Annie Watkins, Elizabeth B. White, Yexin

155 Yang

156 Competing Interests

157 The authors declare no competing interests 
159 Materials and Methods

160 Ethics Statement

161 This study was approved by Yale Human Research Protection Program Institutional Review Boards

162 (FWA00002571, Protocol ID. 2000027690). Informed consents were obtained from all enrolled patients.

163 The healthy blood samples were obtained under the protocol (HIC 0901004619) before the onset of

164 COVID-19 outbreak.

165 Chemicals

166 Tributylamine was purchased from Millipore Sigma. LCMS grade water, methanol, isopropanol,

167 chloroform and acetic acid were purchased through Fisher Scientific. All lipid mediator standards were

168 purchased from Cayman Chemical.

169 Kits and Reagents

170 CBA kits were purchased from BD Biosciences.

171 Patient cohort and serum collection

172 Patients were recruited among those who were admitted to the Yale-New Haven Hospital between

173 March 18th and May 9th, 2020 and were positive for SARS-CoV-2 by RT-PCR from nasopharyngeal

174 and/or oropharyngeal swabs. Patients in this study were enrolled through the IMPACT biorepository

175 study after obtaining informed consent. Basic demographics and clinical information of study

176 participants were obtained and shown in Table 1.

177 Prior to thawing, all samples were gamma-irradiated (2 MRad) to inactivate potential infectious virus.

178 Sample processing for aqueous, organic, and lipid mediator extraction

179 For all LCMS methods LCMS grade solvents were used. Sample order was randomized throughout each

180 extraction. For aqueous and organic metabolites, $50 \mu \mathrm{L}$ patient serum was aliquoted directly into $400 \mu \mathrm{L}$

181 of ice-cold methanol and $500 \mu \mathrm{L}$ of ice-cold chloroform was added. Samples were agitated by shaking for 
18220 minutes at $4{ }^{\circ} \mathrm{C}$ and subsequently centrifuged at $16 \mathrm{kxg}$ for 20 minutes at $4{ }^{\circ} \mathrm{C}$ to induce layering. The

183 top (aqueous) layer and bottom (organic layer) were collected. The aqueous layer was diluted 1:10 in

$18450 \%$ methanol in water and prepared for LCMS injection. The organic layer was taken to dryness in a

185 Savant ${ }^{\mathrm{TM}}$ DNA120 SpeedVac ${ }^{\mathrm{TM}}$ concentrator (Thermo Fisher) and stored at $-80^{\circ} \mathrm{C}$ until analysis. At time of

186 analysis, samples were resuspended in $500 \mu \mathrm{L}$ of $5 \mu \mathrm{g} / \mathrm{mL}$ butylated hydroxytoluene in 6:1

187 isopropanol:methanol and further diluted 1:3 in the same solvent combination for LC-M/MS injection.

\section{Lipid mediators sample processing and extraction}

189 Lipid mediators were extracted from patient serum as previously described. ${ }^{44}$ Briefly $100 \mu \mathrm{L}$ of serum

190 was aliquoted on ice and $1 \mathrm{ng}$ each of d8-5-HETE, d5-RvD2, d5-LXA4, d4-LTB4, d4-PGE2 was added to

191 each sample followed by $400 \mu \mathrm{L}$ of ice-cold methanol. Samples were incubated for $30 \mathrm{~min}$ at $-20^{\circ} \mathrm{C}$ to

192 allow precipitation of protein. Samples were centrifuged at $10 \mathrm{k} \times \mathrm{g}$ for 10 minutes and the supernatant

193 was collected in a fresh tube.

194 Solid phase extraction columns (Sep-Pak ${ }^{\circledR} 3 \mathrm{~mL}, 200 \mathrm{mg}, \mathrm{C} 18$, Waters Corporation) were conditioned in

195 vacuum manifold with $10 \mathrm{~mL}$ of methanol followed by $10 \mathrm{~mL}$ of water. One at a time to each

196 supernatant, $9 \mathrm{~mL}$ of acidified water $(\mathrm{pH} 3.5$ with hydrochloric acid) was added and the samples was

197 quickly loaded onto column. The column was then washed to with $10 \mathrm{~mL}$ of water. Once samples were

198 loaded, columns were washed with $4 \mathrm{~mL}$ of hexanes and then lipid mediators were eluted with $8 \mathrm{~mL}$ of

199 methyl-formate. Samples were dried under nitrogen and resuspended in $100 \mu \mathrm{L}$ of 1:1 water:methanol.

200 For LC-MS analysis $30 \mu \mathrm{L}$ of each sample was injected.

\section{$201 \quad$ LC-MS/MS analysis}

202 Aqueous metabolite, lipid, and lipid mediator samples were analyzed using a series of targeted multiple-

203 reaction monitoring (MRM) methods. All samples were separated using a Sciex ExionLC ${ }^{\mathrm{TM}}$ AC system and 204 analyzed using a Sciex 5500 QTRAP $^{\circledR}$ mass spectrometer. 
Aqueous metabolites were analyzed using a previously established ion pairing method with

206 modification. ${ }^{14}$ Quality control samples were injected after every 10 injections and assessed for signal

207 stability. Samples were separated across a Waters Atlantis T3 column (100Å, $3 \mu \mathrm{m}, 3 \mathrm{~mm} \times 100 \mathrm{~mm})$ and

208 eluted using a binary gradient from $5 \mathrm{mM}$ tributylamine, $5 \mathrm{mM}$ acetic acid in $2 \%$ isopropanol, $5 \%$

209 methanol, $93 \%$ water (v/v) to $100 \%$ isopropanol over 15 minutes. Analytes were detected in negative

210 mode using two distinct MRM pairs for each metabolite when possible. After signal confirmation only

211 one of the MRM signals was taken forward for analysis. Heavy labeled standards were not utilized given

212 the breadth of targets, thus relative quantification was performed. Fidelity of select signals including

213 retention time and spectra was confirmed by comparison to a synthetic molecular reference.

214 Lipid samples were analyzed using a previously established HILIC method with modification. ${ }^{13}$ Samples

215 were separated on a Water XBridge ${ }^{\circledR}$ Amide column $(3.5 \mu \mathrm{m}, 3 \mathrm{~mm} \times 100 \mathrm{~mm})$ and eluted using a 12

216 minute binary gradient from 100\% $5 \mathrm{mM}$ ammonium acetate, 5\% water in acetonitrile apparent pH 8.4

217 to $95 \% 5 \mathrm{mM}$ ammonium acetate, $50 \%$ water in acetonitrile apparent $\mathrm{pH}$ 8.0. Target lipids were

218 detected using scheduled MRM. Lipid signals were divided into two methods utilizing either negative

219 mode or positive mode and a separate injection was analyzed for each method. Both datasets were

220 separately normalized using total-area sum to correct for instrument drift.

221 Lipid mediators were analyzed using a previously established reverse phase method with

222 modifications. ${ }^{44}$ Samples were separated on a Waters Atlantis T3 column (100Å, $3 \mu \mathrm{m}, 3 \mathrm{~mm}$ X $\left.100 \mathrm{~mm}\right)$

223 and using a binary gradient of A: $0.01 \%$ acetic acid in water and B: $0.01 \%$ acetic acid in methanol.

224 Samples were eluted over 20 min from 40-100 \% B. Samples were detected in negative mode using

225 previously published MRM pairs and source conditions. ${ }^{36}$ Triggered spectra were collected using

226 enhanced-product ion scans and rolling collision energy. A blank and a standard mix were serially

227 injected every 10 injections. Standard mix consisted of each of the following compounds at $10 \mathrm{ng} / \mathrm{mL}$ :

228 RvE3, LXA4, LXA5, LXB4, PGE2, PGD2, PGF2a, TxB2, PD1, RvD5, Maresin 1, LTB4, 5,15-DiHETE, 14-HDHA, 
229 18-HEPE, AA, EPA, DHA. Spectra and comparison to authentic standards was used to confirm signal

230 identity.

231 Spectral confirmation was not possible for RvD2, RvD3, LXA5, RvD6, 8,9 DiHETrE, 12-HHT, 11-HETE, 11-

232 HEPE, 7-HDPA, 13-HDPA, 14-HDPA, 17-HDPA, 7-HDHA, 13-HDHA, 17-HDHA and 21-HDHA but identity

233 was assessed by comparison to related standards. These signals were regarded as lower confidence but 234 were used for class comparison of the LMs and multivariate analysis.

235 All signals were integrated using MultiQuant ${ }^{\circledR}$ Software 3.0.3. In total 1,414 molecules were targeted 236 across a water-soluble metabolite method and two organic-extracted lipid methods in either positive or 237 negative ionization modes. Of these 716 features were judged to be positively detected by visual 238 inspection, missing value filtering ( $50 \%$ cut-off) and QC coefficient of variance filtering ( $40 \%$ cut-off after 239 normalization). Remaining missing values were replaced with the minimum group value for that feature.

240 For aqueous and lipid mediator datasets signal quality was judged visually and signal stability was

241 assessed by QC or repeat injection of a standard mix. Lipid mediator data was normalized to internal

242 heavy isotope standards as previously described. ${ }^{44}$

243 Univariate and multivariate analysis was performed in MarkerView ${ }^{\circledR}$ Software 1.3.1. The aqueous

244 dataset and the combined lipid mediators/cytokine dataset data were autoscaled prior to multivariate

245 analysis in order to visualize the contribution of low ionization efficiency species and difference of scales

246 between the cytokine and lipid mediator measurements. Lipid datasets were pareto scaled to avoid

247 overrepresenting low abundance signals within each lipid class. For all univariate analysis an unpaired t-

248 test was used. For univariate analysis of the LM/cytokine set a single moderate group patient was

249 excluded by an extreme studentized deviate test for analysis of PGE2 ( $z=4.58)$.

\section{Quantification of cytokine and chemokine levels}

251 The serum concentration of IFN- $\alpha$, IFN- $\gamma$, IL-1 $\beta$, IL-2, IL-4, IL-6, IL-8, IL-10, IL-12/IL-23p40, IL-17A, MIP-1 $\alpha$,

252 RANTES, TNF- $\alpha$, and MCP-1 was determined using a cytometric bead array according to the 
253 manufacturer's instructions (BD Biosciences). The serum concentration of IL-1 $\alpha$ was determined by an

254 ELISA according to the manufacturer's instructions (R \& D Systems).

\section{Single cell RNA sequencing analysis}

256 The published single cell RNA sequencing dataset from Wilk, et al Nature Medicine 2020 was

257 downloaded from the COVID-19 Cell Atlas (https://www.covid19cellatlas.org/\#wilk20). ${ }^{38}$ Data was read

258 into Seurat v3.0 and each cluster's cellular identity was annotated per Wilk, et al Nature Medicine

$2592020 .^{38}$ Expression levels of ALOX and CYP genes within specific cell types in healthy controls and COVID-

26019 patients was visualized using Seurat's DotPlot feature. ALOX5 expression levels in specific cell types

261 was visualized using the VInPlot feature A Mann-Whitney test was used to determine statistical

262 differences in gene expression between healthy and COVID samples.

\section{Patient Statistics}

264 Demographic data is presented as either counts and percentages (for categorical data) or means and

265 standard deviations (for continuous data). To investigate the difference in the control, moderate and

266 severe groups, GraphPad Prism (version 8.4.2) was used. The results were compared using the chi-

267 square test or Fisher's exact test for categorical variables and one-way analysis of variance (ANOVA) or

268 unpaired t test was used for continuous variables. A p-value of less than 0.05 was considered statistically

269 significant.

270 


\section{$271 \quad$ References}

272 1. Richardson, S., et al. Presenting characteristics, comorbidities, and outcomes among 5700

273 patients hospitalized with COVID-19 in the New York City area. Jama (2020).

274 2. Braverman, N.E. \& Moser, A.B. Functions of plasmalogen lipids in health and disease. Biochimica

275 et Biophysica Acta (BBA)-Molecular Basis of Disease 1822, 1442-1452 (2012).

276 3. Yang, J., et al. Prevalence of comorbidities in the novel Wuhan coronavirus (COVID-19) infection:

277 a systematic review and meta-analysis. International journal of infectious diseases (2020).

278 4. Bellis, C., et al. Human plasma lipidome is pleiotropically associated with cardiovascular risk

279 factors and death. Circulation: Cardiovascular Genetics 7, 854-863 (2014).

280 5. Pietiläinen, K.H., et al. Association of lipidome remodeling in the adipocyte membrane with

281 acquired obesity in humans. PLoS Biol 9, e1000623 (2011).

282 6. Sharma, S., et al. Role of oxidized lipids in pulmonary arterial hypertension. Pulmonary

283 circulation 6, 261-273 (2016).

284 7. Titz, B., et al. Alterations in serum polyunsaturated fatty acids and eicosanoids in patients with

285 mild to moderate chronic obstructive pulmonary disease (COPD). International journal of

286 molecular sciences 17, 1583 (2016).

287 8. Pettersen, D., Davidsson, Ö. \& Whatling, C. Recent advances for FLAP inhibitors. Bioorganic \&

$288 \quad$ Medicinal Chemistry Letters 25, 2607-2612 (2015).

289 9. Blasco, H., et al. The specific metabolome profiling of patients infected by SARS-COV-2 supports

290 the key role of tryptophan-nicotinamide pathway and cytosine metabolism. (Research Square, $2912020)$.

292 10. Shen, B., et al. Proteomic and metabolomic characterization of COVID-19 patient sera. Cell 293 (2020). 
294 11. Troisi, J., et al. Serum metabolomic profile of symptomatic and asymptomatic SARS-CoV-2

295 infected patients. (2020).

296 12. Wu, D., et al. Plasma Metabolomic and Lipidomic Alterations Associated with COVID-19.

297 medRxiv (2020).

298 13. Mackenzie Pearson, S.K.K., Wu, S. \& Baker, P.R. Achieve Broad Lipid Quantitation using a High299 Throughput Targeted Lipidomics Method.

300 14. McCloskey, D., Gangoiti, J.A., Palsson, B.O. \& Feist, A.M. A pH and solvent optimized reversephase ion-paring-LC-MS/MS method that leverages multiple scan-types for targeted absolute quantification of intracellular metabolites. Metabolomics 11, 1338-1350 (2015).

303 15. Dawson, J. \& Walters, M. Uric acid and xanthine oxidase: future therapeutic targets in the prevention of cardiovascular disease? British journal of clinical pharmacology 62, 633-644

16. Hare, J.M. \& Johnson, R.J. Uric acid predicts clinical outcomes in heart failure: insights regarding the role of xanthine oxidase and uric acid in disease pathophysiology. (Am Heart Assoc, 2003).

308 17. Papi, A., et al. Role of xanthine oxidase activation and reduced glutathione depletion in rhinovirus induction of inflammation in respiratory epithelial cells. Journal of Biological

18. Tobin, M.J., Laghi, F. \& Jubran, A. Why COVID-19 Silent Hypoxemia is Baffling to Physicians. American Journal of Respiratory and Critical Care Medicine (2020).

313 19. Chen, X.-Q., Dong, J., Niu, C.-Y., Fan, J.-M. \& Du, J.-Z. Effects of hypoxia on glucose, insulin, glucagon, and modulation by corticotropin-releasing factor receptor type 1 in the rat.

$31620 . \quad$ Newhouse, L.P., et al. Three hours of intermittent hypoxia increases circulating glucose levels in healthy adults. Physiological reports 5, e13106 (2017). 
318 21. Oltmanns, K.M., et al. Hypoxia causes glucose intolerance in humans. American journal of 319 respiratory and critical care medicine 169, 1231-1237 (2004).

320 22. Xie, J., et al. Association between hypoxemia and mortality in patients with COVID-19. in Mayo $321 \quad$ Clinic Proceedings (Elsevier, 2020).

322 23. Reis, A., et al. A comparison of five lipid extraction solvent systems for lipidomic studies of 323 human LDL. Journal of lipid research 54, 1812-1824 (2013).

324 24. Tyagi, R.K., Azrad, A., Degani, H. \& Salomon, Y. Simultaneous extraction of cellular lipids and 325 water-soluble metabolites: evaluation by NMR spectroscopy. Magnetic resonance in medicine 35, 194-200 (1996).

327 25. Lin, C.Y., Wu, H., Tjeerdema, R.S. \& Viant, M.R. Evaluation of metabolite extraction strategies

329 26. Lebrero, P., et al. Cellular plasmalogen content does not influence arachidonic acid levels or 330 distribution in macrophages: A role for cytosolic phospholipase A2 $\gamma$ in phospholipid remodeling. 331 Cells 8, 799 (2019).

332 27. Dennis, E.A. \& Norris, P.C. Eicosanoid storm in infection and inflammation. Nature Reviews 333 Immunology 15, 511-523 (2015).

334 28. Norris, P.C., Gosselin, D., Reichart, D., Glass, C.K. \& Dennis, E.A. Phospholipase A2 regulates 335 eicosanoid class switching during inflammasome activation. Proceedings of the National $336 \quad$ Academy of Sciences 111, 12746-12751 (2014).

337 29. Dennis, E.A., Cao, J., Hsu, Y.-H., Magrioti, V. \& Kokotos, G. Phospholipase A2 enzymes: physical

340 30. Lämmermann, T., et al. Neutrophil swarms require LTB4 and integrins at sites of cell death in $341 \quad$ vivo. Nature 498, 371-375 (2013). 
342 31. Bitto, A., et al. Flavocoxid, a dual inhibitor of COX-2 and 5-LOX of natural origin, attenuates the 343 inflammatory response and protects mice from sepsis. Critical Care 16, R32 (2012).

344 32. Dalli, J. \& Serhan, C.N. Specific lipid mediator signatures of human phagocytes: microparticles 345 stimulate macrophage efferocytosis and pro-resolving mediators. Blood 120, e60-e72 (2012).

346 33. Kowal-Bielecka, O., et al. Cyclooxygenase-and lipoxygenase-derived eicosanoids in 347 bronchoalveolar lavage fluid from patients with scleroderma lung disease: An imbalance

34. Serhan, C.N. \& Haeggstrom, J. Lipid mediators in acute inflammation and resolution: Eicosanoids, PAF, resolvins, and protectins. Fundamentals of Inflammation. CN Serhan, PA Ward, DW Gilroy, and SS Ayoub, editors. Cambridge University Press, Cambridge, 153-174 (2010).

353 35. Serhan, C.N. \& Savill, J. Resolution of inflammation: the beginning programs the end. Nature immunology 6, 1191-1197 (2005).

355 36. Ian Riley, P.C.N., Kathy Luong, Charles Serhan. CET\&RI Spectra Book \& Physical Properties of 356 SPM and Eicosanoids 2019. (2019).

357 37. Tam, V.C., et al. Lipidomic profiling of influenza infection identifies mediators that induce and 358 resolve inflammation. Cell 154, 213-227 (2013).

359 38. Wilk, A.J., et al. A single-cell atlas of the peripheral immune response in patients with severe 360 COVID-19. Nature Medicine, 1-7 (2020).

361 39. Liao, M., et al. Single-cell landscape of bronchoalveolar immune cells in patients with COVID-19. $362 \quad$ Nature medicine, 1-3 (2020).

363 40. Merad, M. \& Martin, J.C. Pathological inflammation in patients with COVID-19: a key role for 364 monocytes and macrophages. Nature Reviews Immunology, 1-8 (2020). 
365 41. Renaud, H.J., Cui, J.Y., Khan, M. \& Klaassen, C.D. Tissue distribution and gender-divergent expression of 78 cytochrome P450 mRNAs in mice. Toxicological sciences 124, 261-277 (2011).

367 42. Huang, C., et al. Clinical features of patients infected with 2019 novel coronavirus in Wuhan, China. The lancet 395, 497-506 (2020).

369 43. Kalinski, P. Regulation of immune responses by prostaglandin E2. The Journal of Immunology 370 188, 21-28 (2012).

371 44. English, J.T., Norris, P.C., Hodges, R.R., Dartt, D.A. \& Serhan, C.N. Identification and profiling of 372 specialized pro-resolving mediators in human tears by lipid mediator metabolomics.

373 Prostaglandins, Leukotrienes and Essential Fatty Acids 117, 17-27 (2017). 
377 Figure 1. Mobilization of plasmalogen-derived PUFAs correlates with the disease severity in COVID-19.

378 (a) Supervised PLSDA analysis of the healthy, moderate and severe disease groups and (b) the

379 corresponding feature loading plot. (c-e) Comparison of moderate to healthy (c), severe to healthy (d)

380 and severe to moderate (e) by unpaired t-test with PUFA classes overlaid. Overlaid data series

381 correspond to lipid species containing at least one copy of C20:4 (blue), C20:5 (orange), C22:5 (purple)

382 or C22:6 (red) acyl chains. (f-h) Comparison of moderate to healthy (f), severe to healthy (g) and severe

383 to moderate (h) by unpaired t-test with plasmalogen lipid series overlaid in red. For (c-h) cutoff lines

384 indicate a positive or negative 2-fold change and a p-value of 0.05 . (i-I) Heatmap of the autoscaled mean

385 intensity of each patient group for significantly varied lipids ( $p<0.05$ ) containing C20:4 (i), C20:5 (j), C22:5

386 (k), and C22:6 (I). Color scale is consistent for (i-I).

388 Figure 2: A unique milieu of LMs defines moderate and severe COVID-19 disease. (a) Unsupervised PCA

389 of autoscaled combined lipid mediator and cytokine dataset and (b) corresponding feature loading plot.

390 (c-e) Univariate comparison of moderate disease to healthy (c), severe disease to healthy (d) and severe

391 to moderate disease (f) by unpaired t-test. Cutoff lines indicate a positive or negative 2-fold change and

392 a p-value of 0.05 . For (b-f) species are colored by class as cytokine (cyan), arachidonic acid-derived (AA-

393 derived, blue), eicosapentaenoic acid-derived (EPA-derived, orange), docosapentaenoic acid-derived

394 (DPA-derived, purple) or docosahexaenoic acid-derived (DHA-derived, red). (f-k) Heatmaps of the

395 autoscaled mean for each patient group across cytokines (f), molecules synthesized by ALOX5 (g),

396 ALOX12 (h), ALOX15 (i), Cyclooxygenases (j) or cytochrome P450 (k). Color scale is consistent across (f-

$397 \mathrm{k})$. 
399 Figure 3. Human PBMCs from COVID-19 patients are enriched for ALOX5 expressing cells and express

400 higher levels of ALOX5. (a) UMAP dimensionality reduction plot of a published human PBMC single-cell

401 RNA Seq dataset (Wilk, et al Nature Medicine 2020) identifying twenty cell types. (b) UMAP depicting

402 ALOX5 expressing cells in blue. (c) Violin plots indicating ALOX5 expression levels within specific cellular

403 populations in healthy (blue) or COVID (red) PBMCs. Statistical significance was determined by a Mann-

404 Whitney test; ${ }^{*} p<0.05 .{ }^{* *} p<0.01$

405

406 
Table 1. Patient demographics, preexisting conditions and treatment distributions. Patient data were

408 compared using the chi-square test or Fisher's exact test for categorical variables and one-way analysis

409 of variance (ANOVA) or unpaired t test was used for continuous variables.

\begin{tabular}{|c|c|c|c|c|}
\hline & $\begin{array}{c}\text { Healthy } \\
(n=19)\end{array}$ & $\begin{array}{l}\text { Moderate } \\
(n=18)\end{array}$ & $\begin{array}{l}\text { Severe } \\
(n=20)\end{array}$ & $p$-value \\
\hline \multicolumn{5}{|l|}{ Demographics } \\
\hline $\begin{array}{l}\text { Mean Age } \pm \text { SD } \\
\text { (Range) }\end{array}$ & $\begin{array}{c}42.7 \pm 14.92 \\
(21-72)\end{array}$ & $\begin{array}{c}60.56 \pm 14.11 \\
(26-92)\end{array}$ & $\begin{array}{c}63.6 \pm 15.17 \\
(32-91)\end{array}$ & $<0.001$ \\
\hline $\begin{array}{l}\text { Mean BMI } \pm \text { SD } \\
\text { (Range) }\end{array}$ & & $\begin{array}{c}29.65 \pm 8.37 \\
(19.49-55.04)\end{array}$ & $\begin{array}{c}31.08 \pm 5.84 \\
(22.87-44)\end{array}$ & 0.5402 \\
\hline Gender, n (\%) & & & & 0.6391 \\
\hline Male & $9(47.37 \%)$ & $11(61.11 \%)$ & $12(60 \%)$ & \\
\hline Female & $10(52.63 \%)$ & 7 (38.89\%) & $8(40 \%)$ & \\
\hline Race, n (\%) & & & & 0.8932 \\
\hline White & 15 (78.95\%) & $13(72.22 \%)$ & $14(70 \%)$ & \\
\hline Black & $2(10.53 \%)$ & 2 (11.11\%) & $3(15 \%)$ & \\
\hline Hispanic & $1(5.26 \%)$ & 2 (11.11\%) & $3(15 \%)$ & \\
\hline Asian & $1(5.26 \%)$ & $1(5.56 \%)$ & $0(0 \%)$ & \\
\hline \multicolumn{5}{|l|}{ Comorbidities } \\
\hline Heart disease & & $5(27.78 \%)$ & $10(50 \%)$ & 0.1983 \\
\hline Hyperlipidemia & & $4(22.22 \%)$ & $6(30 \%)$ & 0.719 \\
\hline Hypertension & & $10(55.56 \%)$ & $15(75 \%)$ & 0.3071 \\
\hline $\begin{array}{r}\text { Chronic lung } \\
\text { diseases }\end{array}$ & & $1(5.56 \%)$ & $4(20 \%)$ & 0.3436 \\
\hline Diabetes & & $5(27.78 \%)$ & $7(35 \%)$ & 0.7342 \\
\hline \multicolumn{5}{|l|}{ Therapies } \\
\hline Tociluzimab & & $9(50 \%)$ & $18(90 \%)$ & 0.0113 \\
\hline Hydroxycloroquine & & $13(72.22 \%)$ & $20(100 \%)$ & 0.0171 \\
\hline Steroids & & $2(11.11 \%)$ & $8(40 \%)$ & 0.0673 \\
\hline Antiviral & & & & 0.5292 \\
\hline Azatanavir & & 11 (61.11\%) & $13(65 \%)$ & \\
\hline Remdesivir trial & & 2 (11.11\%) & $4(20 \%)$ & \\
\hline $\begin{array}{l}\text { Mechanical } \\
\text { ventilation }\end{array}$ & & $0(0 \%)$ & $13(65 \%)$ & $<0.0001$ \\
\hline $\begin{array}{l}\text { Intensive Care } \\
\text { Unit }\end{array}$ & & $0(0 \%)$ & $20(100 \%)$ & $<0.0001$ \\
\hline
\end{tabular}


Figure 1

a

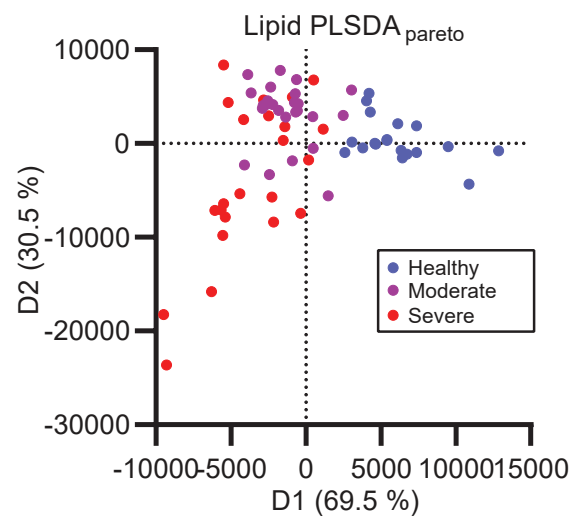

C

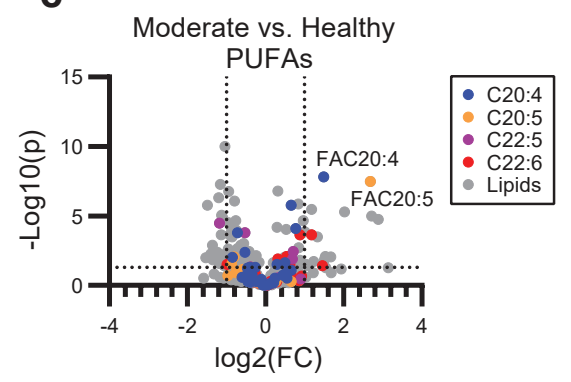

f
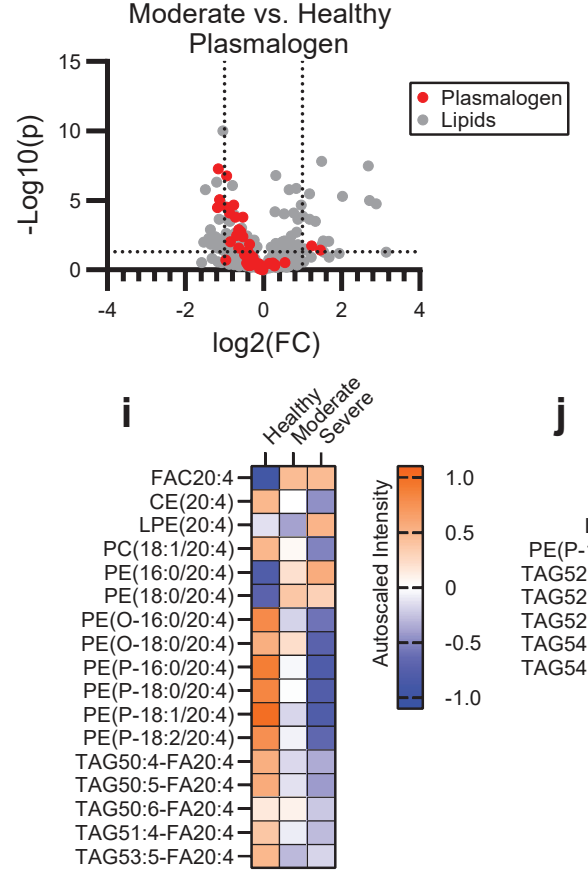

b

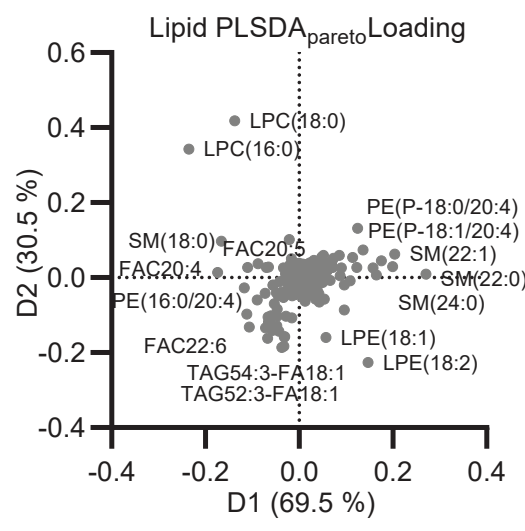

d

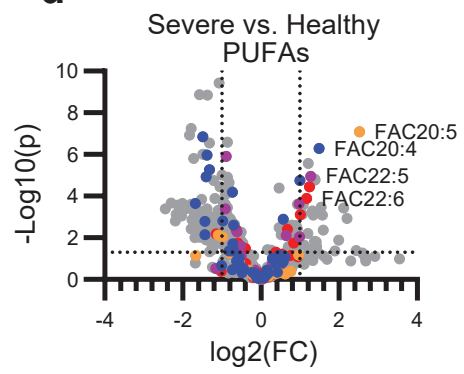

g

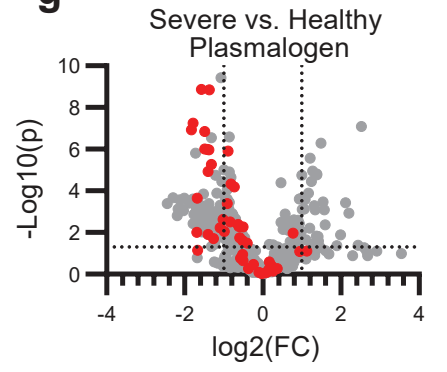

h
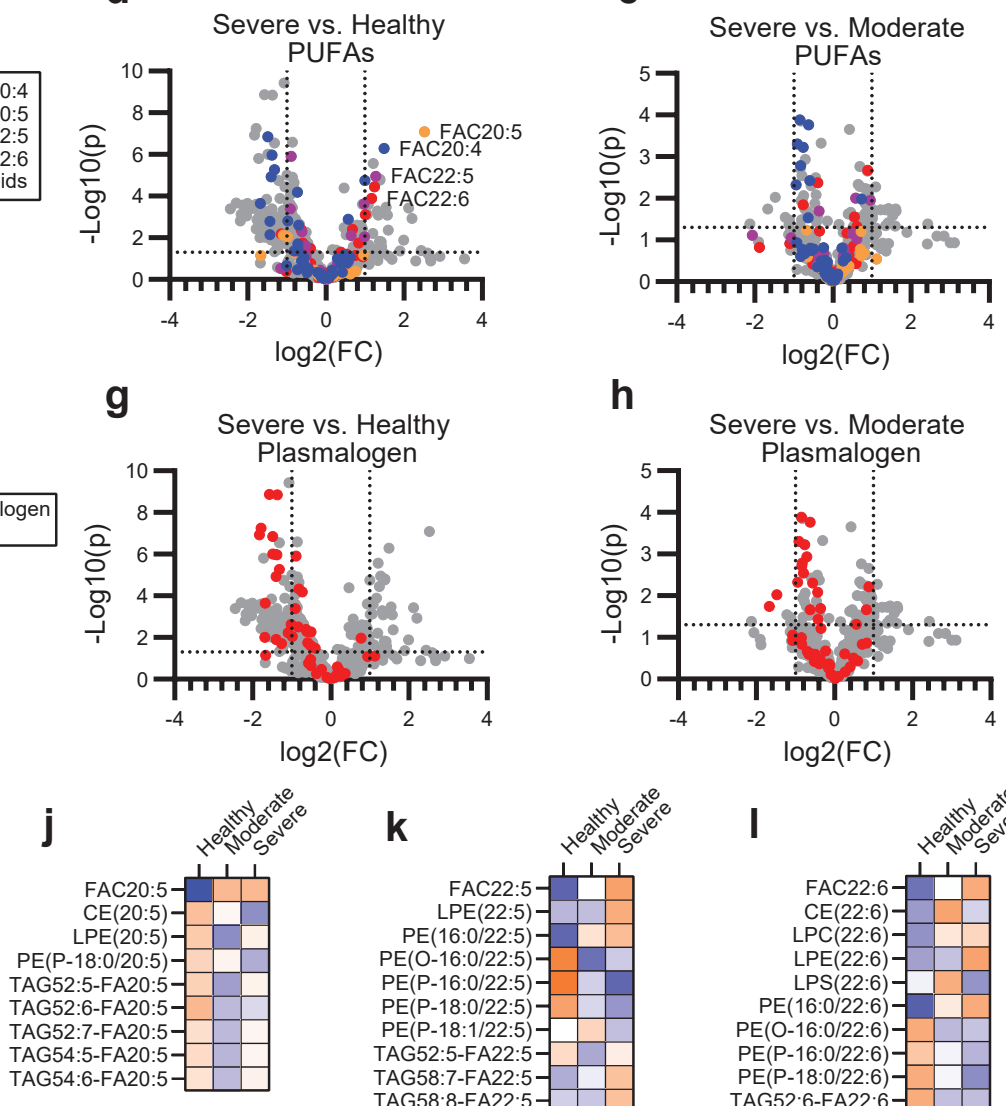

k

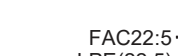
LPE (22:5) PE(16:0/22:5) PE(O-16:0/22:5) PE(P-16:0/22:5) PE(P-18:1/22:5) TAG52:5-FA22:5 TAG58:7-FA22:5 TAG58:8-FA22:5-

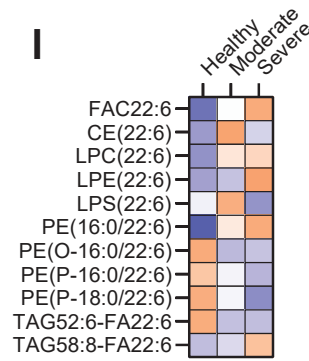


Figure 2

a

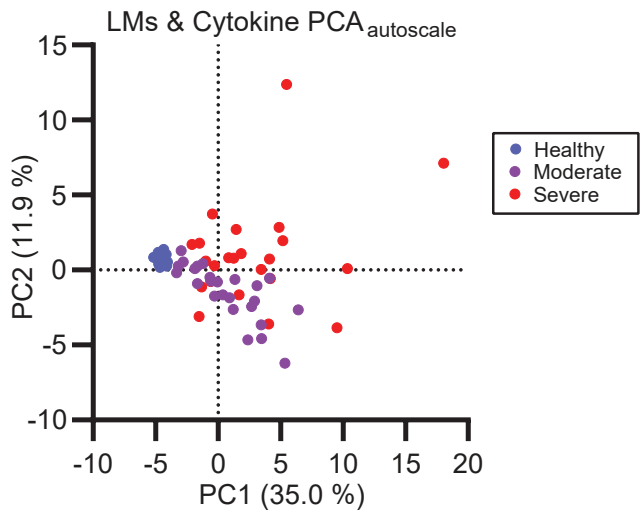

C

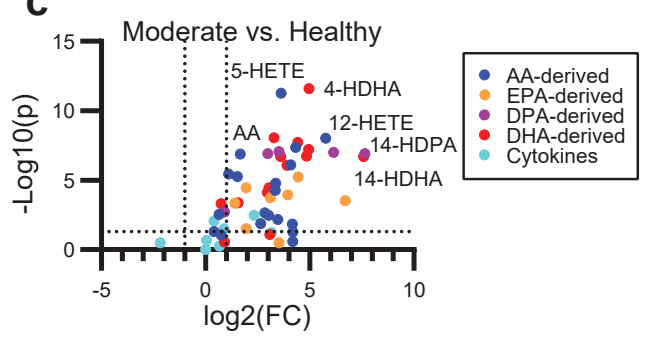

f

Cytokines

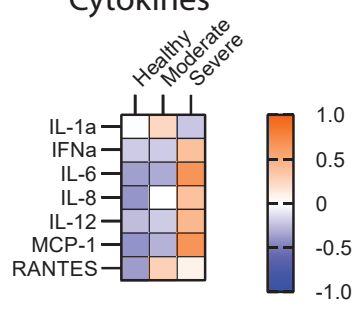

j

Cyclooxygenase

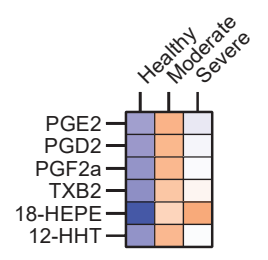

g

k P450 b

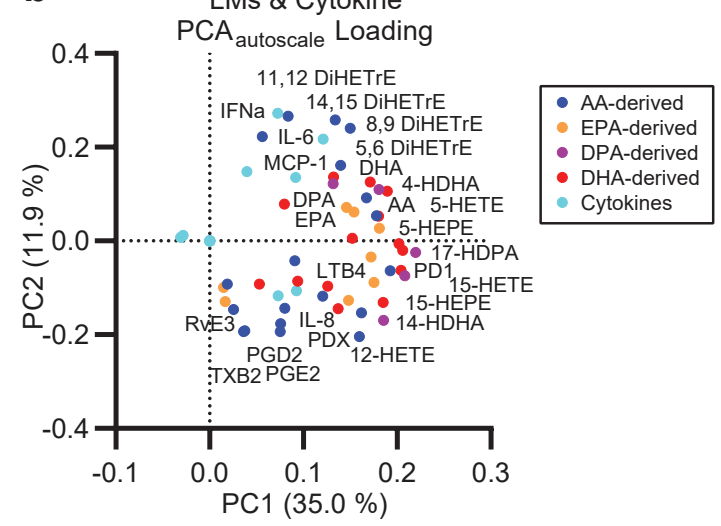

d

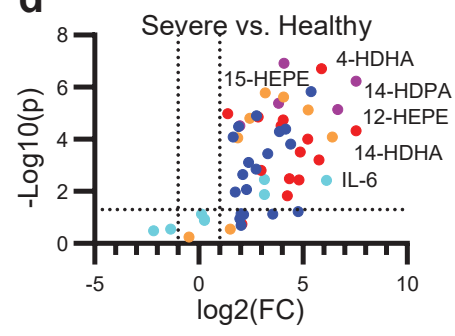

e

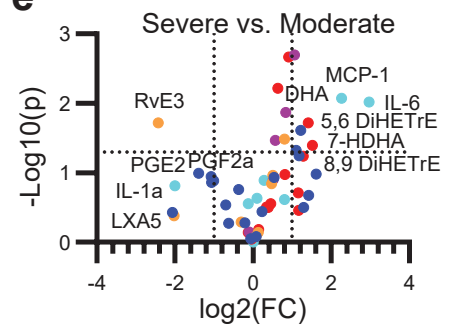

h

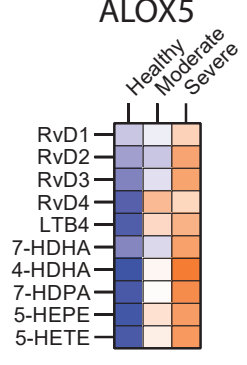

ALOX12
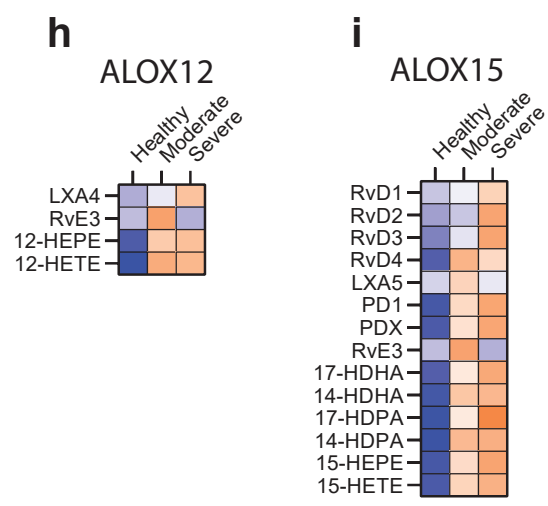

Cytochrome

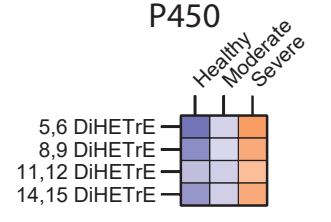


Figure 3

a

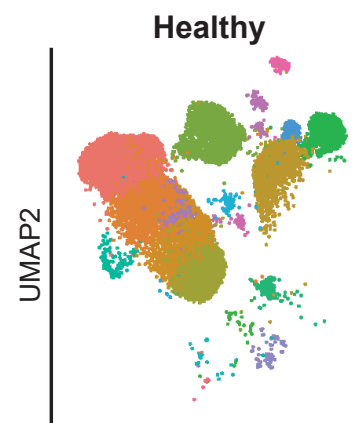

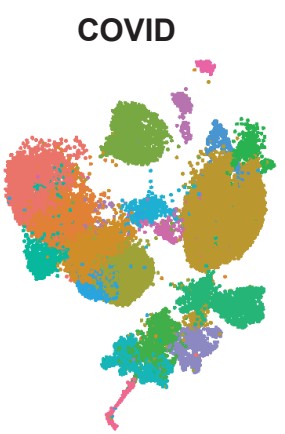

UMAP1
- ${ }^{\mathrm{NK}} \mathrm{CD} 8 \mathrm{~m} \mathrm{~T}$

- CD8m T

CD14 monocyte

- CD4nT

- B

- CD16 monocyte

RBC

Proliferative lymphocyte

- $\lg \mathrm{PB}$

IFN-stim CD4T

- DC

- $\lg A$ PB

- gd T \& eosinophil

- Neutrophil

pDC b

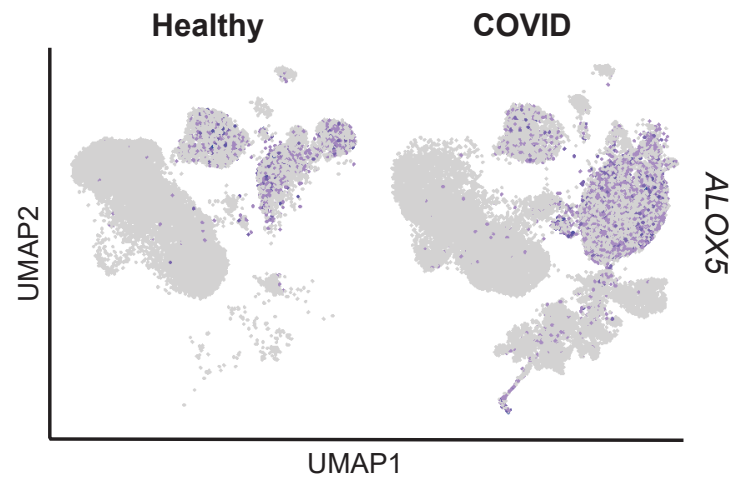

C

CD14 Monocytes

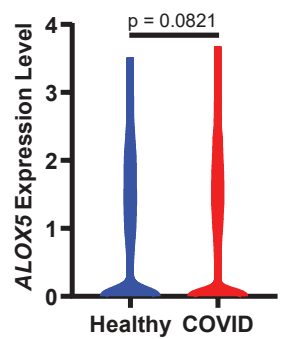

CD16 Monocytes

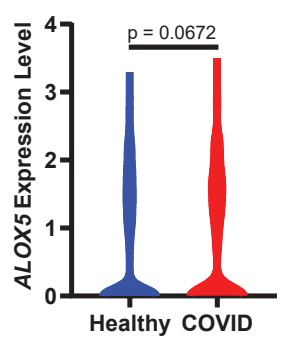

Neutrophils

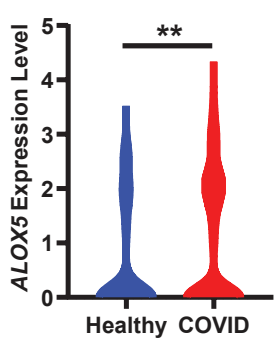

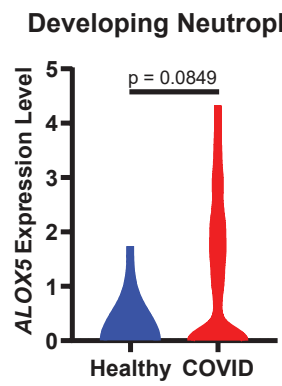

B Cells

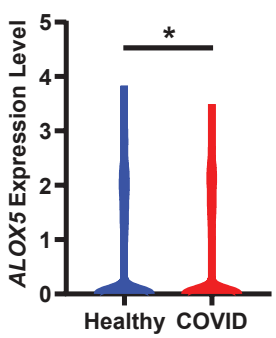

DCs

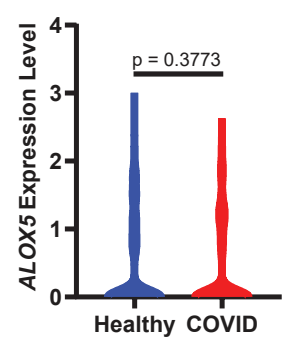




\section{Figures}

Figure 1

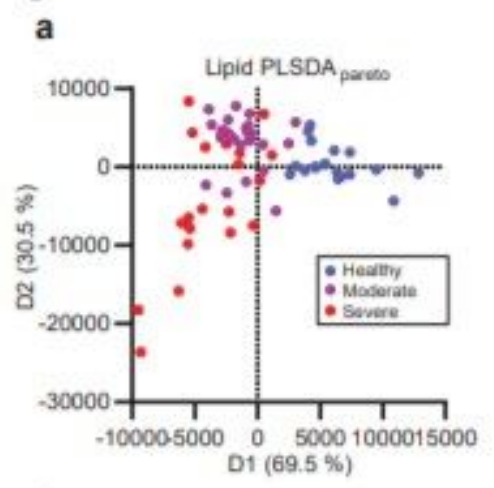

b

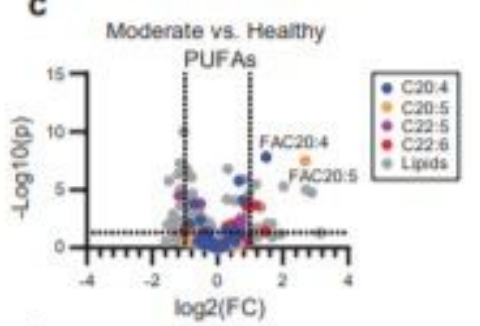

f Moderate vs. Healthy
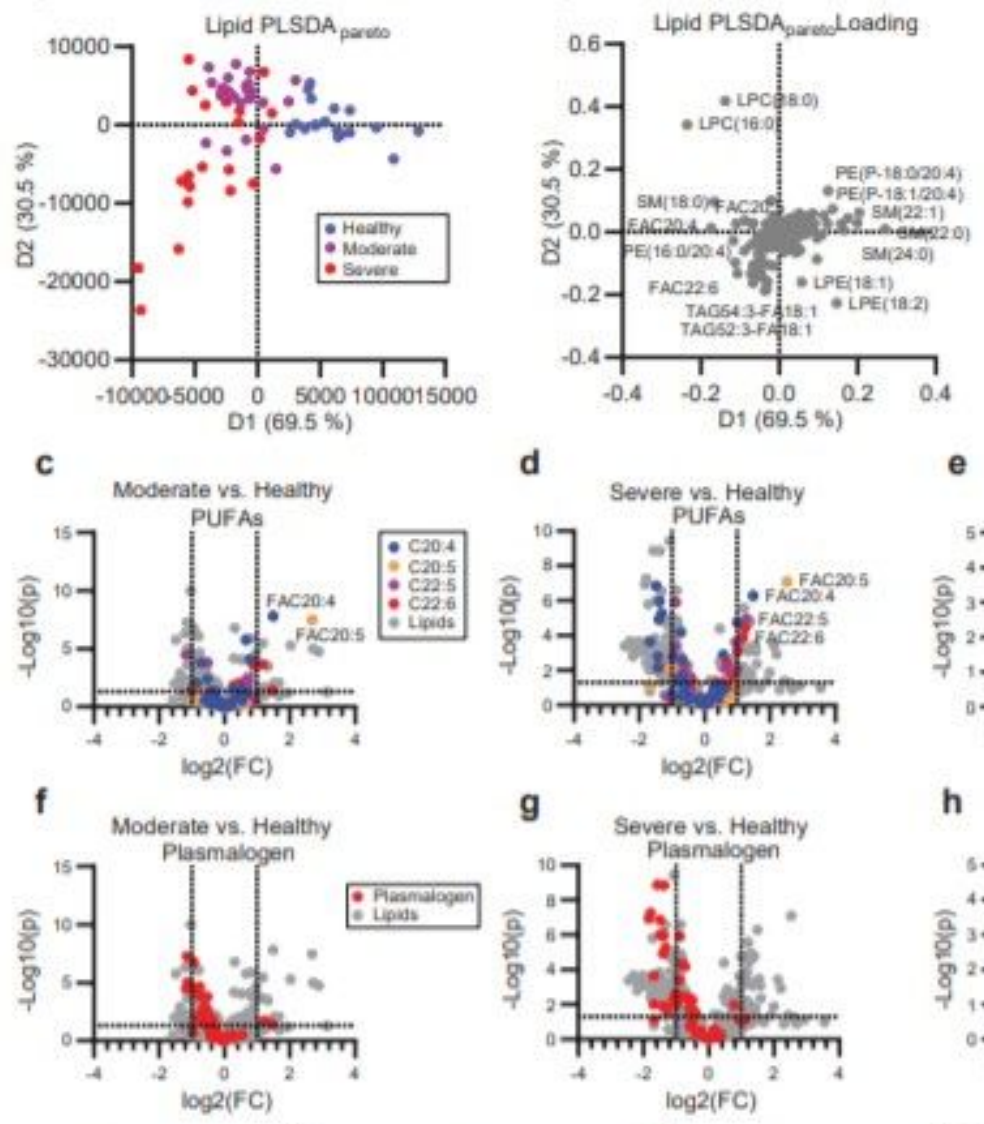

d
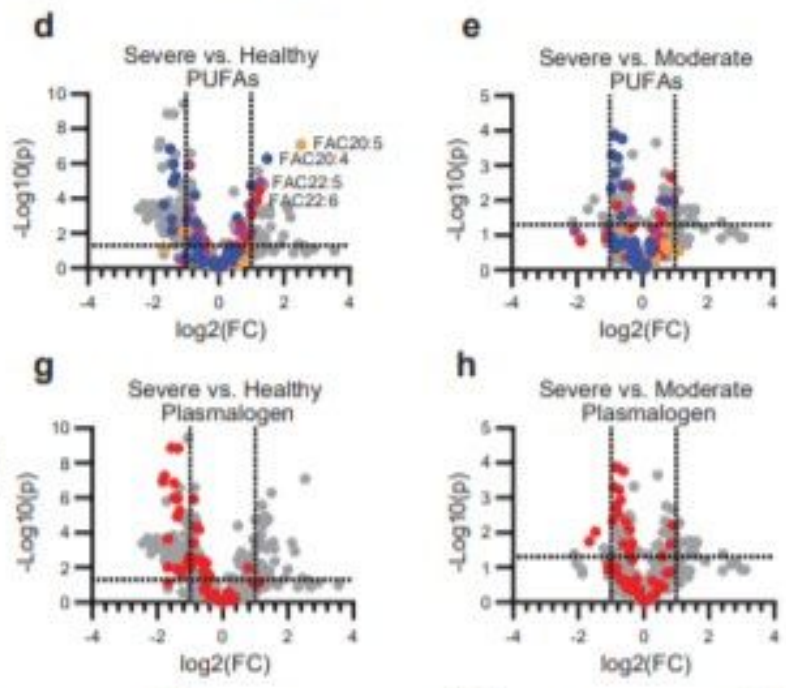

h
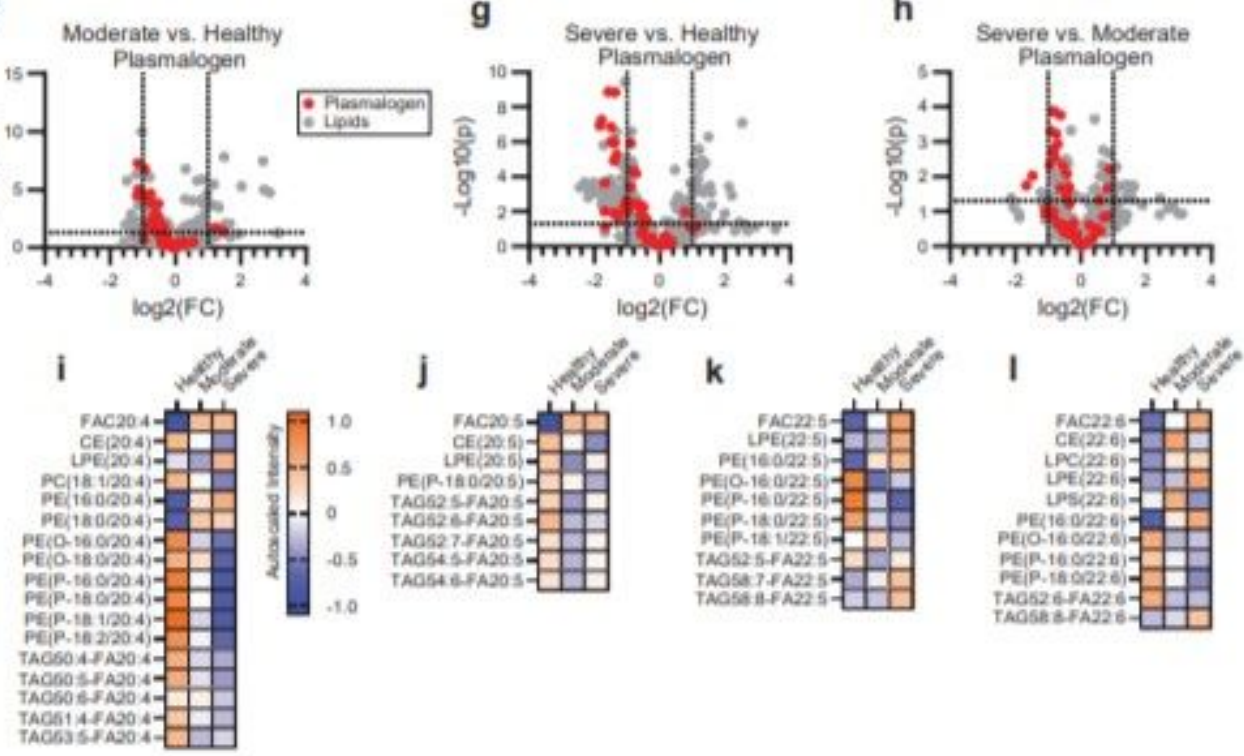

TACDSSTA00 A

\section{Figure 1}

Mobilization of plasmalogen-derived PUFAs correlates with the disease severity in COVID-19. (a) Supervised PLSDA analysis of the healthy, moderate and severe disease groups and (b) the corresponding feature loading plot. (c-e) Comparison of moderate to healthy (c), severe to healthy (d) and severe to moderate (e) by unpaired t-test with PUFA classes overlaid. Overlaid data series correspond to lipid species containing at least one copy of C20:4 (blue), C20:5 (orange), C22:5 (purple) or C22:6 (red) acyl chains. ( $f-h)$ Comparison of moderate to healthy $(f)$, severe to healthy $(g)$ and severe to moderate (h) by unpaired t-test with plasmalogen lipid series overlaid in red. For (c-h) cutoff lines indicate a positive or negative 2-fold change and a p-value of 0.05. (i-I) Heatmap of the autoscaled mean intensity of each 
patient group for significantly varied lipids $(\mathrm{p}<0.05)$ containing C20:4 (i), C20:5 (j), C22:5 (k), and C22:6 (I). Color scale is consistent for (i-l).

Figure 2

a

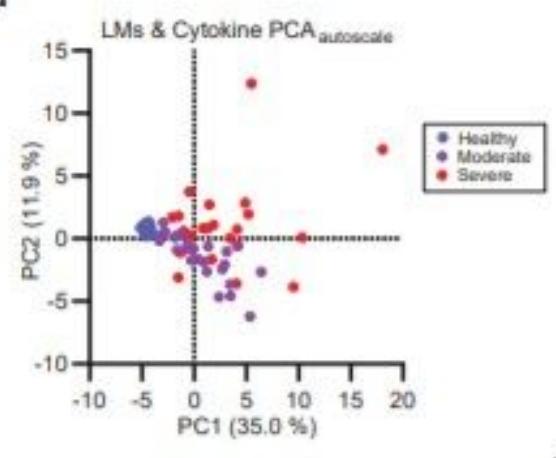

c

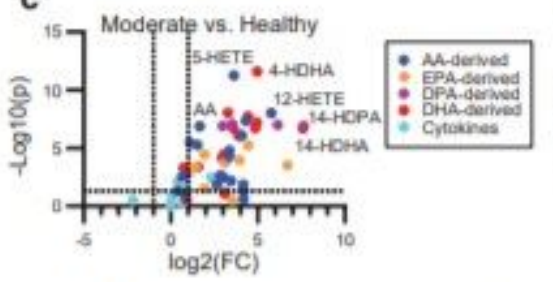

f

Cytokines

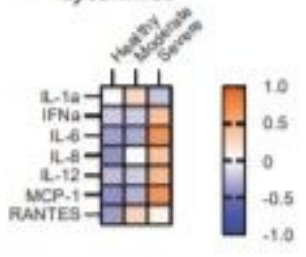

j Cyclooxygenase

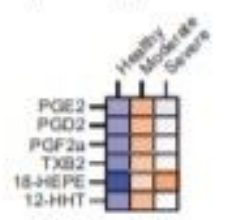

k b LMs \& Cycokine
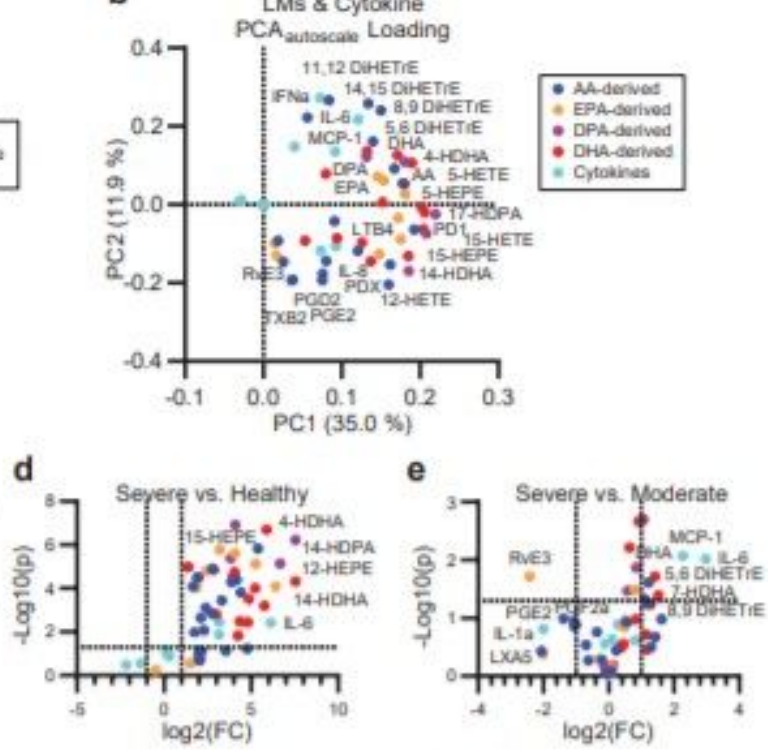

h
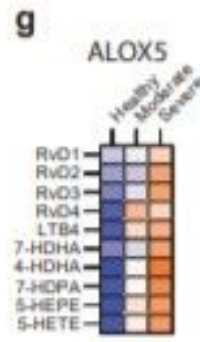

Cytochrome

P450

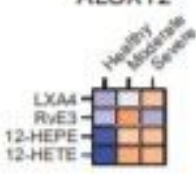

$\mathbf{i}$

ALOXIS

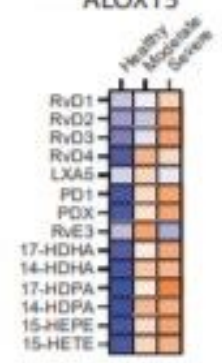

\section{Figure 2}

A unique milieu of LMs defines moderate and severe COVID-19 disease. (a) Unsupervised PCA of autoscaled combined lipid mediator and cytokine dataset and (b) corresponding feature loading plot. (ce) Univariate comparison of moderate disease to healthy (c), severe disease to healthy (d) and severe to 
moderate disease (f) by unpaired t-test. Cutoff lines indicate a positive or negative 2 -fold change and a pvalue of 0.05 . For (b-f) species are colored by class as cytokine (cyan), arachidonic acid-derived (AAderived, blue), eicosapentaenoic acid-derived (EPA-derived, orange), docosapentaenoic acid-derived (DPAderived, purple) or docosahexaenoic acid-derived (DHA-derived, red). ( $f-k)$ Heatmaps of the autoscaled mean for each patient group across cytokines (f), molecules synthesized by ALOX5 (g), ALOX12 (h), ALOX15 (i), Cyclooxygenases (j) or cytochrome P450 (k). Color scale is consistent across (f- k).

Figure 3
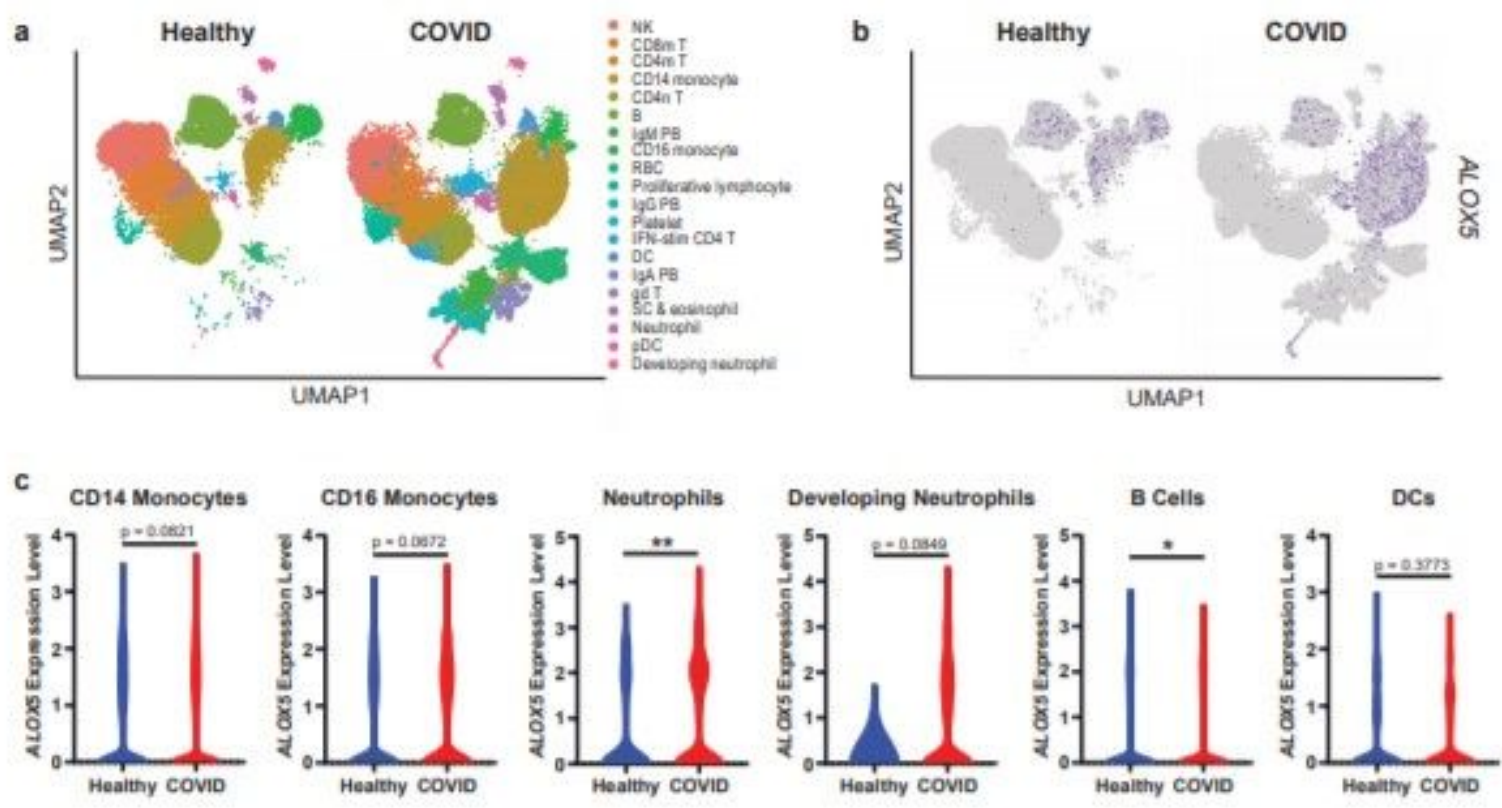

\section{Figure 3}

Human PBMCs from COVID-19 patients are enriched for ALOX5 expressing cells and express higher levels of ALOX5. (a) UMAP dimensionality reduction plot of a published human PBMC single-cell RNA Seq dataset (Wilk, et al Nature Medicine 2020) identifying twenty cell types. (b) UMAP depicting ALOX5 expressing cells in blue. (c) Violin plots indicating ALOX5 expression levels within specific cellular populations in healthy (blue) or COVID (red) PBMCs. Statistical significance was determined by a MannWhitney test; ${ }^{*} p<0.05 .{ }^{* *} p<0.01$.

\section{Supplementary Files}

This is a list of supplementary files associated with this preprint. Click to download. 
- Schwarzet.al.eicosanoidsinCOVID19SupplementaryMaterial.pdf 